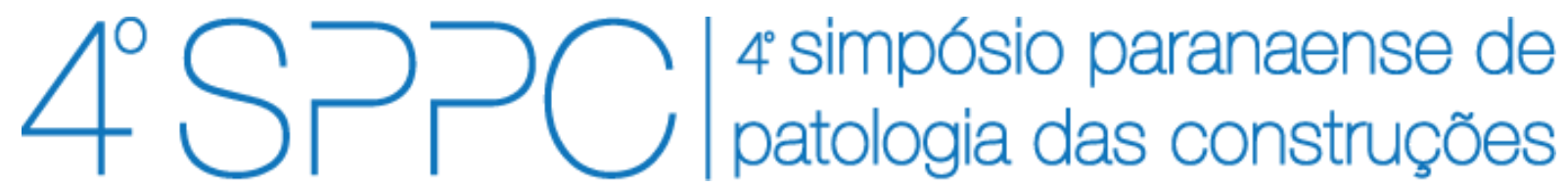

ISSN 2526-7248 artigo n. 4SPPC121, pp. 190-201, 2019

\title{
Localização de danos em vigas de concreto armado considerando o comportamento dos modos de vibração
}

Silva, Matheus Galdino da ${ }^{1}$; Santos, Alexandre Tachibana dos²; Fernandes, João Maurício 3; Gomes, Rodrigo Augusto do Nascimento4; Neri, Marcos Antônio

Teixeira"; Diniz, José Humberto de Oliveira ${ }^{6}$; Jarek, Amanda7; Gavassoni, Elvidio ${ }^{8}$.

${ }^{1}$ Mestrando em Engenharia Civil, PPGECC-UFPR, matheus.silva@lactec.org.br

${ }^{2}$ Engenheiro Civil, Lactec, alexandre.santos@lactec.org.br

${ }^{3}$ Graduando de Engenharia Civil, UFPR, joao.fernandes@lactec.org.br

4, 5,6 Engenheiros Eletricistas, COSERN, marcos.neri@neoenergia.com

7 Dra. em Métodos Numéricos em Engenharia, Lactec, amanda.jarek@lactec.org.br

${ }^{8}$ Dr. em Engenheira Civil, UFPR, gavassoni@ufpr.br

Resumo: O monitoramento da saúde estrutural é um sistema de acompanhamento periódico da integridade aplicado em estruturas civis, onde a análise dinâmica pode ser empregada uma vez que modificações de rigidez são refletidas nos parâmetros modais oscilatórios do sistema. A literatura de interesse aponta que a análise de correlação dos modos de vibração - com o emprego dos coeficientes MAC e COMAC - nem sempre é capaz de detectar satisfatoriamente a ocorrência e a localização de danos. O presente estudo, contudo, ao inferir sobre a qualidade dos dados utilizados para definição desses coeficientes e sobre etapas posteriores de análise, pretende sugerir a eficâcia da localização de danos, principalmente com o emprego do coeficiente COMAC. As análises foram desenvolvidas utilizando o software computacional de elementos finitos Ansys, para uma viga de concreto armado de pequeno porte. Foram analisados 4 casos de deterioração física com perda de concreto em parte da seção transversal do elemento. Mostra-se que tal abordagem é eficaz na localização de danos com significativa precisão, a depender da discretização dos dados avaliados e do pós processamento matemático aplicado.

Palavras-chave: Ensaios não destrutivos, Análise modal, Integridade estrutural.

Abstract: Structural Health Monitoring is a system used to periodically assess the integrity of civil structures, where modal dynamic analysis may be applied to detect stiffness differences through oscillatory modal parameters. Interest literature suggests that the correlation analysis of the mode shapes - using coefficients such as MAC and COMAC - is not always capable of satisfactorily detecting and locating damage. The present study, however, inferring about the used quality data to define these coefficients and about further analysis steps, intends to suggest the efficiency of locating damage, mainly considering the COMAC coefficient. By means of finite element analysis, using the computational software Ansys, modal analyses were developed to a small size reinforced concrete beam. Losses of concrete on the cross sections due to physical deterioration were considered in 4 different cases. It is shown that this approach has significant accuracy in locating damage, depending on the discretization of the evaluated data and on the usage of post processing mathematical steps.

Keywords: Non-destructive testing, Modal analysis, Structural integrity. 


\section{Introdução} DOI: $10.4322 / 2526-7248.041$

Estruturas de concreto armado, ao longo da sua vida útil, ficam expostas a diferentes processos físicos e químicos de deterioração onde a redução da capacidade resistente desses elementos pode extrapolar os estados limites de segurança da mesma. Quando tais estágios de deterioração se apresentam, tanto riscos a vidas humanas bem como perdas financeiras são resultados indesejados da utilização da estrutura comprometida. Recentemente, algumas companhias privadas de fornecimento de energia elétrica têm registrado incidentes em postes de concreto armado pertencentes às suas redes de distribuição, os quais têm apresentado falhas durante a execução de manutenção periódica nas linhas. Tais eventos têm motivado o crescente interesse na aplicação de técnicas de avaliação periódica deste tipo de elemento, visando minimizar a ocorrência destes.

O processo contínuo de identificação de danos é conhecido como Monitoramento da Saúde Estrutural (do inglês Structural Health Monitoring - SHM), cujo objetivo é determinar o nível de integridade, em qualquer momento da vida útil, considerando tanto uma região localizada como toda a peça analisada. As diretrizes centrais para a aplicação de um sistema SHM envolvem o desenvolvimento de ensaios e medições constantes, além da extração dos dados de interesse e a elaboração de análises estatísticas, de modo a prever o nível de integridade de uma dada estrutura [1]. Além da análise visual, são empregados também ensaios destrutivos e não destrutivos para avaliar elementos e construções civis. Haja vista as dificuldades de execução periódica de ensaios destrutivos, ensaios não destrutivos se apresentam como alternativa viável para a aplicação de um sistema SHM.

Um dos ensaios não destrutivos mais conhecidos é o baseado na Análise Modal Estrutural [2], onde o diagnóstico sobre danos e fissuras, incluindo a posição e a magnitude dos mesmos, é obtido pela variação dos parâmetros modais oscilatórios que refletem as modificações estruturais provenientes dos processos de degradação. Em geral, para análise dinâmica modal, embasada em estudos realizados em condições e ambiente controlados, tal abordagem se mostra como uma ferramenta capaz de auxiliar na aplicação do SHM em problemas da Engenharia Civil [3-4].

Atualmente, a literatura de interesse apresenta a avaliação da correlação de diferentes modos de vibração, com o uso dos coeficientes MAC e COMAC, como um método nem sempre eficaz para a localização de danos em uma estrutura [5-7] ao avaliar isoladamente o valor desses coeficientes. Contudo, o objetivo do presente estudo é abordar uma maneira diferente de avaliar o comportamento de tal parâmetro modal, avaliando principalmente a quantidade de dados disponíveis nos autovetores e as descontinuidades noticiadas na função do coeficiente COMAC, para localizar danos em vigas de concreto armado. Com o emprego do software computacional de elementos finitos Ansys, foi modelada a estrutura de interesse e, posteriormente, foram inseridos danos localizados no concreto em distintas posições da mesma, configurando diferentes casos de análise. Os resultados apresentados são baseados na comparação das condições de deterioração física com o mesmo elemento em sua condição íntegra, para o coeficiente COMAC e também na análise exclusiva do primeiro modo de vibração. Nos dois casos foram aplicadas etapas de pós processamento matemático, com conceitos de taxa de variação, para potencializar a localização dos danos implementados na peça analisada. 
SILVA, M. G.; SANTOS, A. T.; FERNANDES, J. M.; GOMES, R. A. N.; NERI, M. A. T.; DINIZ, J. H.; JAREK, A.; ELVIDIO, G. LOCALIZAÇÃO DE DANOS EM VIGAS DE CONCRETO ARMADO CONSIDERANDO O COMPORTAMENTO DOS MODOS DE VIBRAÇÃO. $4^{\circ}$ Simpósio Paranaense de Patologia das Construções (40 SPPC), artigo 4SPPC121, pp. 190 - 201, 2019.

\section{Localização de danos} DOI: $10.4322 / 2526-7248.041$

\subsection{Modos de vibração}

Os coeficientes MAC (Modal Assurance Criterion) e COMAC (Coordinate Modal Assurance Criterion) são critérios matemáticos, baseados na análise dos modos de vibração de uma estrutura, utilizados para detectar alterações no comportamento modal da mesma e, consequentemente, avaliar o seu nível de deterioração [7]. O coeficiente MAC, também conhecido como um coeficiente de correlação, apresenta uma análise de dispersão em linha reta entre dois conjuntos de dados. No cálculo desse coeficiente, se obtém um resultado único para a peça, uma vez que o processo está fundamentado na soma de todos os graus de liberdade considerados. Por outro lado, para o coeficiente COMAC são preservados os valores individuais nesta soma, onde cada um se refere a um grau de liberdade específico [2]. Matematicamente, os coeficientes MAC e COMAC são definidos por:

$$
M A C=\frac{\left|\sum_{j=1}^{n}\left(\psi_{A}\right)_{j}\left(\psi_{B}\right)_{j}^{*}\right|^{2}}{\left(\sum_{j=1}^{n}\left(\psi_{A}\right)_{j}\left(\psi_{A}\right)_{j}^{*}\right)\left(\sum_{j=1}^{n}\left(\psi_{B}\right)_{j}\left(\psi_{B}\right)_{j}^{*}\right)} \quad(01) \quad \operatorname{COMAC}_{(i)}=\frac{\left[\sum_{j=1}^{m} \mid\left[\psi_{A}\right]_{i}^{j}\left[\psi_{B}\right]_{i}^{j}\right]^{2}}{\sum_{j=1}^{m}\left(\left[\psi_{A}\right]_{i}^{j}\right)^{2} \sum_{j=1}^{m}\left(\left[\psi_{B}\right]_{i}^{j}\right)^{2}}
$$

onde: $\psi_{A}$ e $\psi_{B}$ representam, respectivamente, os j-ésimos vetores dos modos de vibrar de um elemento íntegro e do mesmo elemento deteriorado para $i$ pontos ao longo do seu comprimento. Além disso, as variáveis destacadas com "*" são os mesmos vetores apresentados anteriormente, porém transpostos.

O coeficiente COMAC difere do coeficiente MAC à medida que aquele busca apresentar informações locais mesmo combinando dados de diferentes modos de vibração [4]. Desse modo, o COMAC é utilizado para identificar em qual região os modos de vibração da estrutura não são correlatos (análise proveniente do coeficiente MAC) e, consequentemente, onde se encontram regiões danificadas [6-7], onde a variação em relação ao índice igual a 1,0 pode ser interpretada como o nível de denificação da estrutura, com valores até 0,9 apresentando correlação satisfatória [2].

\subsection{Taxa de variação}

A taxa de variação média de uma curva $f(x)$ qualquer, em um ponto específico, é dependente da inclinação da reta secante, definida pelas coordenadas desse ponto e do ponto seguinte no qual passa a reta [8]. Nesse sentido, para a reta secante essa taxa é definida pela Equação 03. Ademais, a distância entre dois pontos $\left(\Delta_{x}\right)$ define a qualidade da reta secante considerada, onde a menor distância leva a melhor aproximação. Ao levar esse processo de aproximação ao limite $\left(\Delta_{x} \rightarrow 0\right)$, define-se 0 conceito de reta tangente como uma linha que intercepta dada curva em apenas um ponto [9]. Como não é possível igualar o denominador $\left(\Delta_{x}\right)$ da Equação 03 a zero, surge o conceito de limites e a taxa de variação instantânea é dada pela Equação 04.

$\frac{f(x+\Delta x)-f(x)}{\Delta x}$

$$
\lim _{\Delta x \rightarrow 0} \frac{f(x+\Delta x)-f(x)}{\Delta x}
$$

O limite apresentado na Equação 04 é a derivação de $f(x)$ no ponto $x$, desde de que o limite exista e seja finito. Quando o limite de uma função não existe ela é dita não derivável e é identificada em situações com tangentes verticais, descontinuidades na função ou em mudanças bruscas na inclinação da curva [8]. 
SILVA, M. G.; SANTOS, A. T.; FERNANDES, J. M.; GOMES, R. A. N.; NERI, M. A. T.; DINIZ, J. H.; JAREK, A.; ELVIDIO, G. LOCALIZACCÃO DE DANOS EM VIGAS DE CONCRETO ARMADO CONSIDERANDO O COMPORTAMENTO DOS MODOS DE VIBRAÇÃO. $4^{\circ}$ Simpósio Paranaense de Patologia das Construções (40 SPPC), artigo 4SPPC121, pp. 190 - 201, 2019.

\section{Modelo computacional} DOI: $10.4322 / 2526-7248.041$

Para o desenvolvimento do modelo e da análise computacional foi selecionado o software de Elementos Finitos (EF) Ansys Workbench (versão 18.2). Esse software foi escolhido devido à sua capacidade de processar análises dinâmicas e também

pela diversidade de elementos disponíveis para a modelagem estrutural [10]. A estrutura modelada possui dimensões de $10 \mathrm{~cm} \times 10 \mathrm{~cm} \times 140 \mathrm{~cm}$, armada com 4 barras de aço longitudinais de $6,3 \mathrm{~mm}$ de diâmetro nominal e 14 estribos de 4,2 mm

de diâmetro nominal espaçados a cada $10 \mathrm{~cm}$. As propriedades geométricas e físicas da viga, com base na condição íntegra, estão detalhadas na Figura 1 e na

Tabela 1.
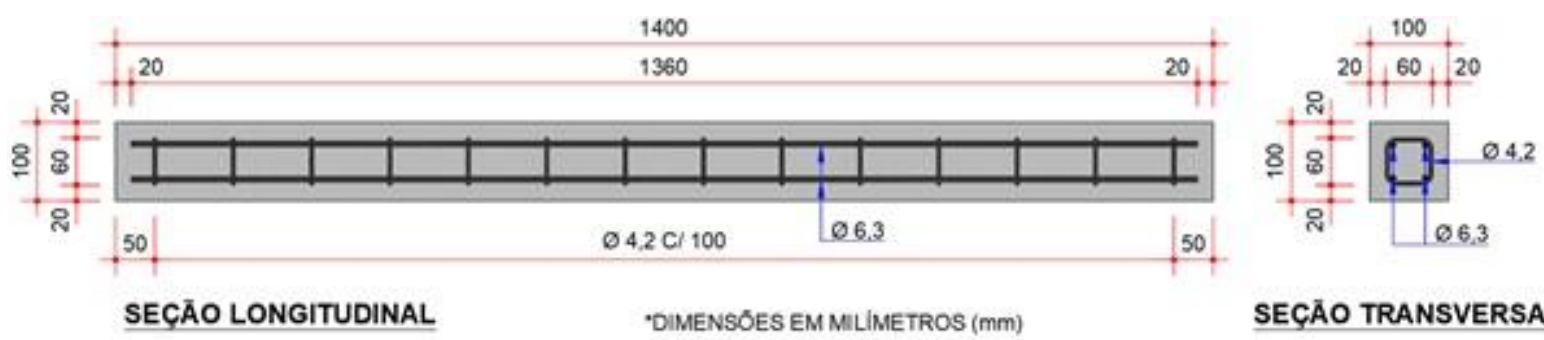

SEÇÃO TRANSVERSAL

Figura 1: Projeto da viga de concreto armado considerada.

Tabela 1: Propriedades físicas da viga de concreto armado considerada.

\begin{tabular}{c|c|c|c|c|c}
\hline \multicolumn{3}{c|}{ Concreto } & \multicolumn{3}{c}{ Aço Estrutural } \\
\hline $\begin{array}{c}\text { Módulo de } \\
\text { Elasticidade }\end{array}$ & $\begin{array}{c}\text { Coef. de } \\
\text { Poisson }\end{array}$ & Densidade & $\begin{array}{c}\text { Módulo de } \\
\text { Elasticidade }\end{array}$ & $\begin{array}{c}\text { Coef. de } \\
\text { Poisson }\end{array}$ & Densidade \\
\hline $40 \mathrm{GPa}$ & 0,20 & $2.400 \mathrm{~kg} / \mathrm{m}^{3}$ & $200 \mathrm{GPa}$ & 0,30 & $7.850 \mathrm{~kg} / \mathrm{m}^{3}$ \\
\hline
\end{tabular}

Para modelar computacionalmente o concreto armado, foram utilizados elementos finitos tridimensionais do tipo SOLID187 (na opção tetraédrica) para representar o concreto e elementos finitos unidimensionais do tipo BEAM188 (na forma quadrática) para representar a armadura [11]. Para simular uma aderência perfeita entre esses componentes, a malha de EF foi desenvolvida com coincidência nodal. Além disso, o tamanho máximo desses elementos foi definido em $5 \mathrm{~mm}$, de modo a permitir uma resolução satisfatória para a análise de interesse, garantindo uma distância máxima entre nós de 2,5 mm. A aplicação dessa malha levou a um modelo com 146.142 nós e 95.073 elementos, conforme a discretização ilustrada na Figura 2.

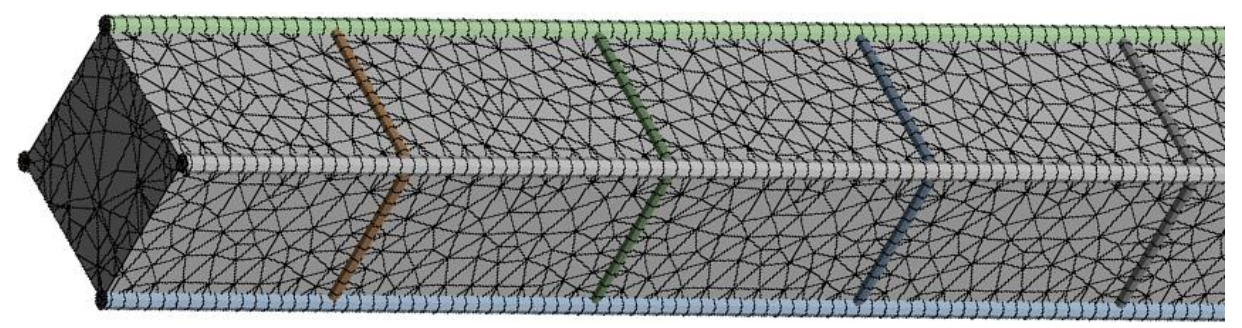

Figura 2: Discretização da estrutura de concreto armado para a análise em FE. 
Como condição de contorno, adotou-se um corpo sem restrições ao movimento (livrelivre), excluindo as incertezas referentes aos parâmetros de apoio. Para obtenção dos autovalores (frequências) e autovetores (modos de vibração), foi utilizado como método de extração o algoritmo Block Lanczos, que permite a resolução de problemas para matrizes simétricas de grandes proporções por meio da utilização de blocos de vetores conforme apresentado por Grimes e Simon [12].

\section{Metodologia}

A representatividade do modelo computacional proposto foi validada com base na análise de dados computacionais comparados com resultados experimentais e analíticos, conforme discutido por Jarek et al. [13]. Com a representatividade do modelo assegurada, foi possível desenvolver as análises de interesse do presente estudo. Em relação à estrutura íntegra apresentada no item 3, foram desenvolvidos 4 novos modelos computacionais implementando-se um dano induzido (considerado no concreto) em diferentes posições, onde cada modelo define um caso a ser avaliado. O dano imposto na viga possui 1 milímetro de espessura no sentido longitudinal e ocupa metade da seção transversal da mesma, conforme exemplificado na Figura 3.
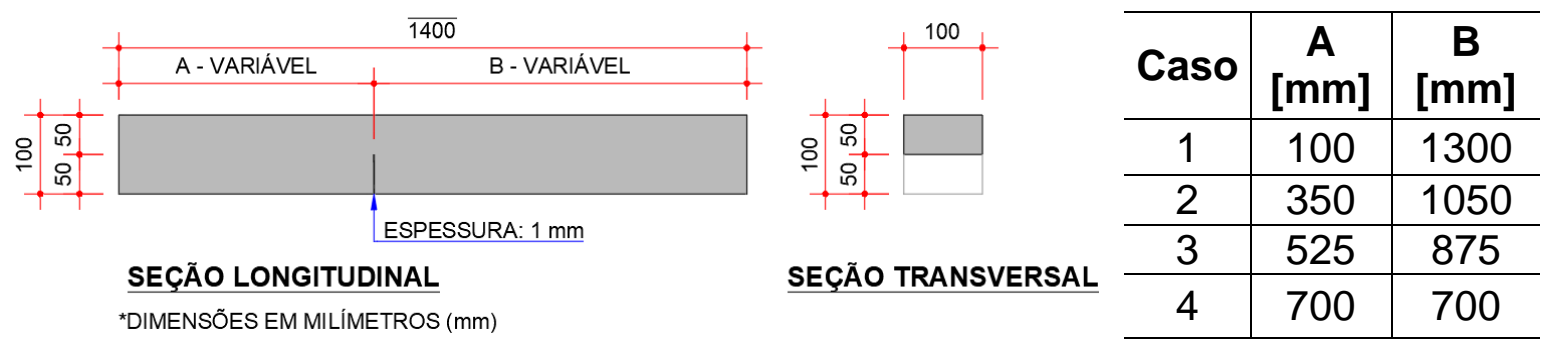

Figura 3: Geometria do dano induzido no modelo computacional e valores das variáveis $A$ e $B$ em cada caso estabelecido.

Ao avaliar o comportamento dinâmico da estrutura como um corpo livre, foram extraídos do modelo computacional os dados das cinco primeiras frequências naturais transversais e os modos de vibração a elas associados, para os quatro casos considerados além da condição íntegra. Como a seção transversal do protótipo é retangular com lados iguais, o comportamento modal do elemento é computacionalmente idêntico nas duas direções. Assim, nesse estudo foram analisadas apenas as propriedades modais no sentido de menor inércia para seção transversal localizada no dano, ou seja, na qual a flexão causa a abertura das fissuras.

Com os autovetores dos modos de vibração para as situações propostas foram calculados, por meio das Equações 01 e 02, os coeficientes de correlação MAC e COMAC para cada caso estabelecido. Além disso, foi aplicado um pós processamento de dados, com base na Equação 3, para a análise do comportamento dos coeficientes COMAC encontrados. Com base na taxa de variação secante (de primeira e de segunda ordem) da curva desses coeficientes, foram então localizados (de forma gráfica e analítica) os danos implementados nos modelos computacionais. O conceito de taxa de variação secante foi aplicado também diretamente ao conjunto de dados do primeiro modo de vibração de cada caso proposto, com o intuito a avaliar 
SILVA, M. G.; SANTOS, A. T.; FERNANDES, J. M.; GOMES, R. A. N.; NERI, M. A. T.; DINIZ, J. H.; JAREK, A.; ELVIDIO, G. LOCALIZAÇÃO DE DANOS EM VIGAS DE CONCRETO ARMADO CONSIDERANDO O COMPORTAMENTO DOS MODOS DE

VIBRAÇÃO. 40 Simpósio Paranaense de Patologia das Construções (40 SPPC), artigo 4SPPC121, pp. 190 - 201, 2019. DOI: $10.4322 / 2526-7248.041$

a capacidade de identificação de danos sobre esses dados, excluindo assim a necessidade da comparação de correlação com a dados da estrutura íntegra.

\section{Resultados e discussão}

Nessa seção são apresentados os resultados principais obtidos no estudo, bem como são registrados os resultados secundários de interesse. Inicialmente, foram extraídos os cinco modos de vibração selecionados tanto para a viga íntegra (Figura 4) bem como para os 4 casos de deterioração estabelecidos no item 4.

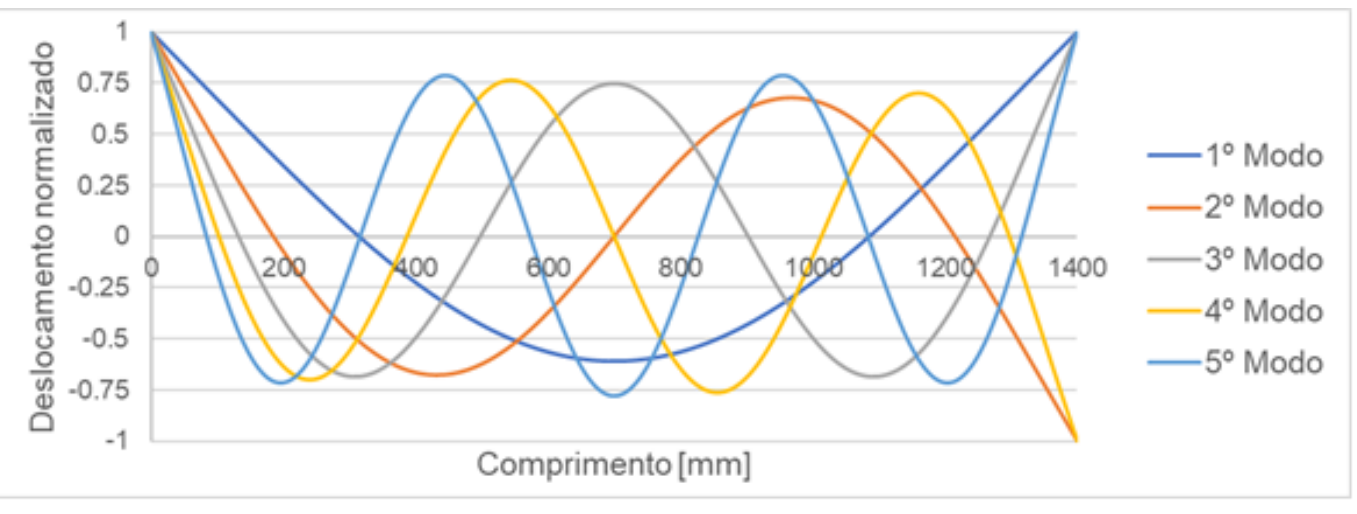

Figura 4: Exemplo dos modos de vibrar transversais normalizados da viga íntegra.

Os autovetores (modos de vibração) são apresentados com comportamento normalizado representativo, uma vez que não foram consideradas taxas de amortecimento no modelo numérico. Além disso, a extração de dados, em cada caso, foi realizada para 4 diferentes resoluções de espaçamento entre pontos (ao longo do comprimento da estrutura), para avaliar a influência da discretização dos dados extraídos do modelo computacional. Foram consideradas resoluções $(\Delta x)$ de $28 \mathrm{~mm}$; $7 \mathrm{~mm} ; 3,5 \mathrm{~mm}$ e $1,75 \mathrm{~mm}$.

Destaca-se que a variação das resoluções propostas não muda o comportamento global dos modos de vibração analisados. Por outro lado, com menores resoluções e maiores conjuntos de dados, o objetivo é avaliar a influência, na localização dos danos, da discretização dos resultados extraídos do modelo computacional para um mesmo comportamento dinâmico da estrutura.

Os coeficientes MAC obtidos para as 16 combinações possíveis (4 casos vs. 4 resoluções) são apresentados no lado esquerdo da Tabela 2. Nota-se que todos os valores do referido coeficiente estão acima de 0,98 , indicando uma correlação significativa entre os modos de vibração das vigas danificadas em relação a viga íntegra e, consequentemente, pouca probabilidade de haver danos na estrutura. Além disso, o lado direito da 
SILVA, M. G.; SANTOS, A. T.; FERNANDES, J. M.; GOMES, R. A. N.; NERI, M. A. T.; DINIZ, J. H.; JAREK, A.; ELVIDIO, G. LOCALIZAÇÃO DE DANOS EM VIGAS DE CONCRETO ARMADO CONSIDERANDO O COMPORTAMENTO DOS MODOS DE VIBRAÇÃO. $4^{\circ}$ Simpósio Paranaense de Patologia das Construções (40 SPPC), artigo 4SPPC121, pp. 190 - 201, 2019. DOI: $10.4322 / 2526-7248.041$

Tabela 2 aponta as cinco primeiras frequências naturais relacionadas aos modos de vibração em cada caso de dano analisado, não sendo observadas variações significativas (variação máxima de 10\%) para o mesmo modo de vibração. 
SILVA, M. G.; SANTOS, A. T.; FERNANDES, J. M.; GOMES, R. A. N.; NERI, M. A. T.; DINIZ, J. H.; JAREK, A.; ELVIDIO, G. LOCALIZAÇÃO DE DANOS EM VIGAS DE CONCRETO ARMADO CONSIDERANDO O COMPORTAMENTO DOS MODOS DE VIBRAÇÃO. 40 Simpósio Paranaense de Patologia das Construções (40 SPPC), artigo 4SPPC121, pp. 190 - 201, 2019. DOI: $10.4322 / 2526-7248.041$

Tabela 2: Valores do coeficiente MAC e das frequências para os diferentes casos.

\begin{tabular}{|c|c|c|c|c|c|c|c|c|c|c|}
\hline \multirow{2}{*}{\multicolumn{2}{|c|}{$\Delta \mathrm{x}[\mathrm{mm}]:$}} & \multicolumn{4}{|c|}{ MAC } & \multicolumn{5}{|c|}{ Frequências [Hz] } \\
\hline & & 28 & 7 & 3,5 & 1,75 & Modo 1 & Modo 2 & Modo 3 & Modo 4 & Modo 5 \\
\hline \multirow{4}{*}{$\begin{array}{l}\text { \&్ల } \\
\text { ర్ }\end{array}$} & 1 & 0,984 & 0,983 & 0,983 & 0,983 & 211,86 & 564,05 & 1051,5 & 1630,8 & 2255,0 \\
\hline & 2 & 0,991 & 0,990 & 0,990 & 0,990 & 204,61 & 520,00 & 999,4 & 1655,7 & 2335,9 \\
\hline & 3 & 0,994 & 0,994 & 0,994 & 0,994 & 194,80 & 535,90 & 1059,0 & 1613,2 & 2325,7 \\
\hline & 4 & 0,996 & 0,996 & 0,996 & 0,996 & 190,49 & 565,26 & 990,1 & 1667,8 & 2261,3 \\
\hline
\end{tabular}

O coeficiente COMAC, assim como o MAC, apresentou valores bastante próximos a 1,0, conforme demonstrado na Figura 5 (a, b, $g$ \& h) para o caso 2 (dano em $A=$ $350 \mathrm{~mm})$, mesmo com diferentes valores de resolução $(\Delta x)$ dos dados analisados. Ademais, avaliando esses gráficos COMAC e as recomendações disponíveis na literatura [2, 5-7] conclui-se que o dano está localizado próximo da região de $A=550 \mathrm{~mm}$ da viga, uma vez que é a região de menor valor do índice. Por outro lado, nota-se na região de indução do dano no modelo computacional $(A=350 \mathrm{~mm})$ uma descontinuidade significativa no comportamento da curva COMAC, noticiada principalmente na Figura $5(\mathrm{~b}, \mathrm{~g} \& \mathrm{~h})$ - para melhores resoluções de $\Delta x$.

Ao aplicar os conceitos de taxa de variação secante (TV) de primeira e de segunda ordem ao conjunto de dados da Figura 5 (a, b, g \& h), comprova-se que os danos não estão localizados necessariamente nos pontos onde o coeficiente COMAC apresenta menor valor, mas sim nas regiões de maior mudança na taxa de variação. Para a resolução de $28 \mathrm{~mm}$, com taxas de variação apresentadas na Figura 5 (c \& e) - para TV de primeira e segunda ordem respectivamente - a localização gráfica não se mostrou eficiente. Porém, a partir da resolução de $7 \mathrm{~mm}$ os resultados gráficos para localização do dano são mais efetivos, como apresentado para a resolução de $7 \mathrm{~mm}$ na Figura 5 (d \& f), para a resolução de $3,5 \mathrm{~mm}$ na Figura 5 (i \& k) e para a resolução de $1,75 \mathrm{~mm}$ na Figura 5 (j \& I).

Além da análise gráfica, na Tabela 3 são apresentadas as posições obtidas para a localização de danos por meio do coeficiente COMAC, para todos os casos considerados, e seus respectivos erros. Nas duas análises (gráfica e analítica), notase que quanto melhor é a discretização do conjunto de dados do modo de vibração (menores valores de $\Delta x$ ), melhor é a precisão sobre a localização da região danificada. Além disso, analiticamente se mostra mais efetivo considerar a taxa de variação de segunda ordem em relação a taxa de variação de primeira ordem. Apesar de não serem identificados resultados satisfatórios com $\Delta x=28 \mathrm{~mm}$, nas duas abordagens apresentadas, a partir de $\Delta x=7 \mathrm{~mm}$ a localização do dano é realizada de maneira mais satisfatória, com variações inferiores a $1,5 \mathrm{~cm}$, considerando a taxa de variação de segunda ordem para o pós-processamento de dados ou a avaliação dos gráficos de taxa de variação, independentemente da ordem considerada. Ressalta-se ainda que a localização de danos independe da posição do mesmo na estrutura, uma vez que o comportamento dos resultados obtidos é similar nos quatro casos avaliados nesse estudo. 
SILVA, M. G.; SANTOS, A. T.; FERNANDES, J. M.; GOMES, R. A. N.; NERI, M. A. T.; DINIZ, J. H.; JAREK, A.; ELVIDIO, G. LOCALIZACCÃO DE DANOS EM VIGAS DE CONCRETO ARMADO CONSIDERANDO O COMPORTAMENTO DOS MODOS DE VIBRAÇÃO. $4^{\circ}$ Simpósio Paranaense de Patologia das Construções (40 SPPC), artigo 4SPPC121, pp. 190 - 201, 2019. DOI: $10.4322 / 2526-7248.041$

(a)
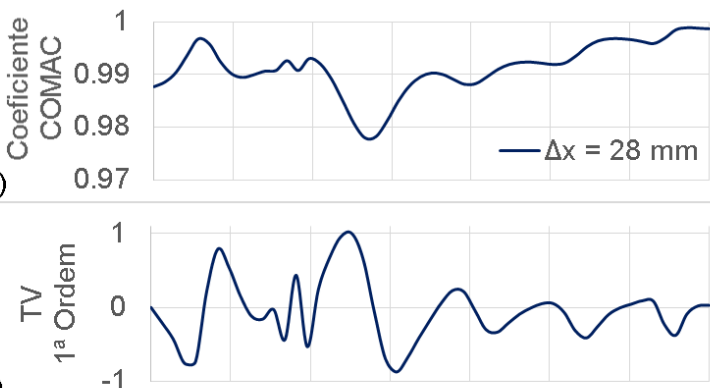

(c)

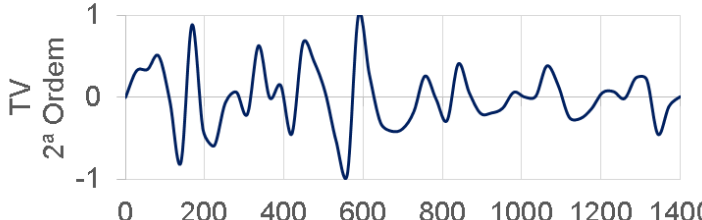

(e)

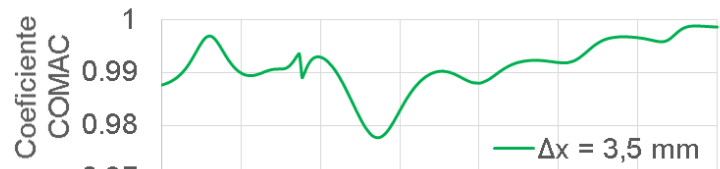

(g)

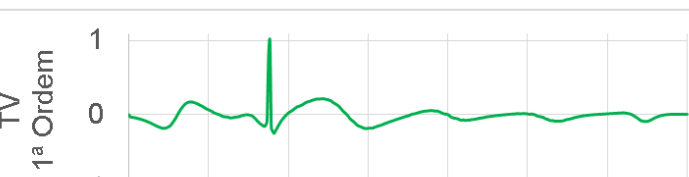

(i)

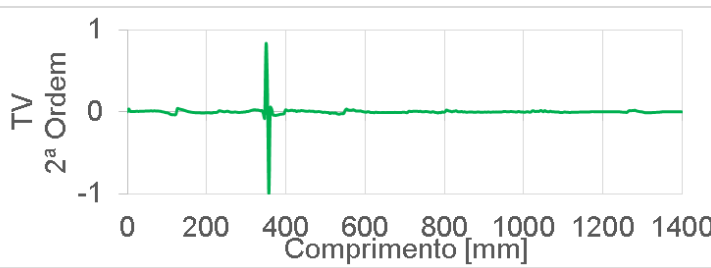

(b)

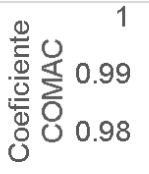

(b) $\quad 0.97$

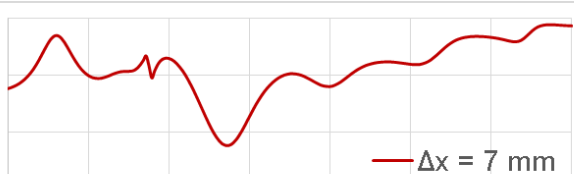

$-\Delta \mathrm{x}=7 \mathrm{~mm}$



(d)
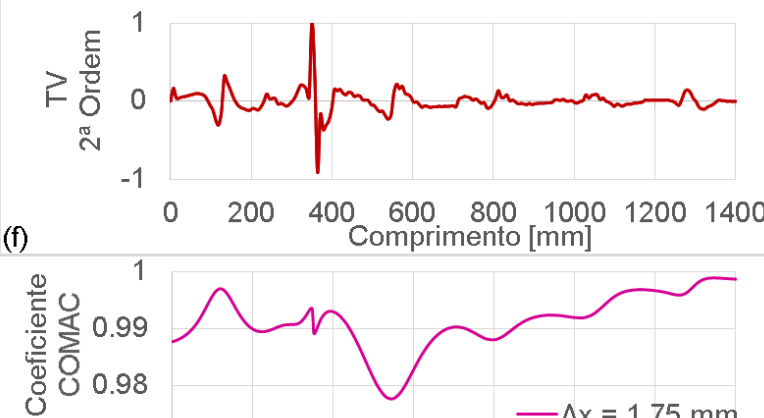

(h)
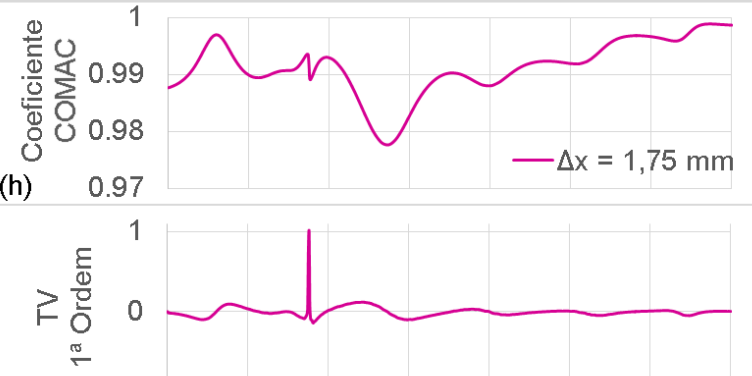

(j)



Figura 5: Coeficientes COMAC ((a), (b), (g) \& (h)); e taxas de variação de $1^{\text {a }}((\mathrm{c}),(\mathrm{d})$ (i) \& (j)), e $2^{\mathrm{a}}((\mathrm{e}),(\mathrm{f}),(\mathrm{k}) \&(\mathrm{l}))$ ordens, para resoluções com $\Delta x$ de $28 \mathrm{~mm}, 7 \mathrm{~mm}$, $3,5 \mathrm{~mm}$ e $1,75 \mathrm{~mm}$ no caso 2 de deteriorção $(A=350 \mathrm{~mm})$.

Tabela 3: Localização do dano por meio das taxas de variação de $1^{\underline{a}}$ e $2^{a}$ ordens do coeficiente COMAC.

\begin{tabular}{|c|c|c|c|c|c|c|c|c|}
\hline \multirow[b]{3}{*}{ Caso } & \multicolumn{8}{|c|}{ Localização do dano para TV de $1^{\text {a }}$ ordem para dados COMAC } \\
\hline & \multicolumn{2}{|c|}{$\Delta x=28 \mathrm{~mm}$} & \multicolumn{2}{|c|}{$\Delta x=7 \mathrm{~mm}$} & \multicolumn{2}{|c|}{$\Delta x=3,5 \mathrm{~mm}$} & \multicolumn{2}{|c|}{$\Delta \mathrm{x}=1,75 \mathrm{~mm}$} \\
\hline & Estimado & Erro & Estimado & Erro & Estimado & Erro & Estimado & Erro \\
\hline 1 & 140,00 & $40 \%$ & 98,00 & $-2 \%$ & 101,50 & $2 \%$ & 99,75 & $0 \%$ \\
\hline 2 & & $44 \%$ & & $2 \%$ & & $1 \%$ & & $1 \%$ \\
\hline 3 & 588 & $12 \%$ & & $-79 \%$ & & $-79 \%$ & & $-80 \%$ \\
\hline \multirow[t]{3}{*}{4} & 812,00 & $16 \%$ & 798,00 & $14 \%$ & 798,00 & $14 \%$ & 796,25 & $14 \%$ \\
\hline & \multicolumn{8}{|c|}{ Localização do dano para TV de $2^{\mathrm{a}}$ ordem para dados COMAC } \\
\hline & $\Delta x=28$ & $\mathrm{~mm}$ & $\Delta x=7$ & & $\Delta x=3,5$ & $\mathrm{~mm}$ & $\Delta x=1,75$ & $\mathrm{~mm}$ \\
\hline Caso & Estimado & Erro & Estimado & Erro & Estimado & Erro & Estimado & Erro \\
\hline 1 & 112,00 & $12 \%$ & 112,00 & $12 \%$ & 101,50 & $2 \%$ & 103,25 & $3 \%$ \\
\hline 2 & 112,00 & $-68^{c}$ & 364 & $4 \%$ & & $2 \%$ & & $1 \%$ \\
\hline 3 & 168,00 & $-68 \%$ & 525,00 & $0 \%$ & 528 & $1 \%$ & 526,75 & $0 \%$ \\
\hline 4 & 868,00 & $24 \%$ & 700,00 & $0 \%$ & 700,00 & $0 \%$ & 703,50 & $1 \%$ \\
\hline
\end{tabular}


SILVA, M. G.; SANTOS, A. T.; FERNANDES, J. M.; GOMES, R. A. N.; NERI, M. A. T.; DINIZ, J. H.; JAREK, A.; ELVIDIO, G. LOCALIZAÇÃO DE DANOS EM VIGAS DE CONCRETO ARMADO CONSIDERANDO O COMPORTAMENTO DOS MODOS DE VIBRAÇÃO. 40 Simpósio Paranaense de Patologia das Construções (40 SPPC), artigo 4SPPC121, pp. 190 - 201, 2019. DOI: $10.4322 / 2526-7248.041$

Como transcorrido no item 4, o conceito de taxa de variação secante foi também aplicado exclusivamente ao conjunto de dados do primeiro modo de vibração, considerando os diferentes casos de implementação do dano na viga. Na Figura 6 é possível visualizar as descontinuidades geradas nas curvas de taxa de variação tanto de primeira como de segunda ordem. Os resultados gráficos dessa análise, principalmente na Figura 6 (a \& c), mostram que essa análise pode ser mais eficiente na detecção de danos considerando resoluções maiores ( $\Delta x$ de $28 \mathrm{~mm}$ ), em relação aos dados COMAC, independente da taxa de variação considerada. Além disso, para resoluções com melhor discretização - como apresentado na Figura 6 (b \& d) para $\Delta \mathrm{x}=7 \mathrm{~mm}$, na Figura 6 (e \& g) para $\Delta \mathrm{x}=3,5 \mathrm{~mm}$ e na Figura 6 (f \& $\mathrm{h}$ ) para $\Delta \mathrm{x}=1,75$ $\mathrm{mm}$ - a eficiência na localização dos danos se manteve equiparada nos dois métodos avaliados.

Por outro lado, a Tabela 4 - onde são apresentadas as posições obtidas para a localização de danos por meio do primeiro modo de vibração, para todos os casos considerados, e seus respectivos erros - ressalta a relevância de considerar a taxa de variação de segunda ordem para uma identificação efetiva do dano, com baixo erro percentual e sem a necessidade de emprego de meios gráficos. A despeito dos resultados encontrados, não é recomendável a utilização direta dos dados de modo de vibração em detrimento do coeficiente COMAC, uma vez que o dano pode, eventualmente, estar localizado sobre o nó do respectivo modo de vibração analisado, comprometendo a capacidade de detecção do mesmo.

Tabela 4: Localização dos danos por meio das taxas de variação de $1^{\underline{a}}$ e $2^{\underline{a}}$ ordens para $01^{\circ}$ modo de vibração.

\begin{tabular}{|c|c|c|c|c|c|c|c|c|}
\hline \multirow[b]{3}{*}{ Caso } & \multicolumn{8}{|c|}{ Localização do dano para TV de $1^{\text {a }}$ ordem para dados do $1^{\circ}$ modo } \\
\hline & \multicolumn{2}{|c|}{$\Delta x=28 \mathrm{~mm}$} & \multicolumn{2}{|c|}{$\Delta \mathrm{x}=7 \mathrm{~mm}$} & \multicolumn{2}{|c|}{$\Delta x=3,5 \mathrm{~mm}$} & \multicolumn{2}{|c|}{$\Delta \mathrm{x}=1,75 \mathrm{~mm}$} \\
\hline & Estimado & Erro & Estimado & Erro & Estimado & Erro & Estimado & Erro \\
\hline 1 & 84,00 & $-16 \%$ & 98,00 & $-2 \%$ & 101,50 & $2 \%$ & 99,75 & $0 \%$ \\
\hline 2 & 84,00 & $-76 \%$ & 28,00 & $-92 \%$ & 24,50 & $-93 \%$ & 3,50 & $-99 \%$ \\
\hline 3 & 84,00 & $-84 \%$ & 70,00 & $-87 \%$ & 7,00 & $-99 \%$ & 1,75 & $-100 \%$ \\
\hline \multirow[t]{3}{*}{4} & 84,00 & $-88 \%$ & 63,00 & $-91 \%$ & 59,50 & $-92 \%$ & 3,50 & $-100 \%$ \\
\hline & \multicolumn{8}{|c|}{ Localização do dano para TV de $2^{a}$ ordem para dados do $1^{\circ}$ modo } \\
\hline & \multicolumn{2}{|c|}{$\Delta x=28 \mathrm{~mm}$} & \multicolumn{2}{|c|}{$\Delta \mathrm{x}=7 \mathrm{~mm}$} & \multicolumn{2}{|c|}{$\Delta \mathrm{x}=3,5 \mathrm{~mm}$} & \multicolumn{2}{|c|}{$\Delta \mathrm{x}=1,75 \mathrm{~mm}$} \\
\hline Caso & Estimado & Erro & Estimado & Erro & Estimado & Erro & Estimado & Erro \\
\hline 1 & 112,00 & $12 \%$ & 112,00 & $12 \%$ & 105,00 & $5 \%$ & 103,25 & $3 \%$ \\
\hline 2 & 392,00 & $12 \%$ & 357,00 & $2 \%$ & 353,50 & $1 \%$ & 350,00 & $0 \%$ \\
\hline 3 & 560,00 & $7 \%$ & 532,00 & $1 \%$ & 528,50 & $1 \%$ & 525,00 & $0 \%$ \\
\hline 4 & 728,00 & $4 \%$ & 700,00 & $0 \%$ & 700,00 & $0 \%$ & 700,00 & $0 \%$ \\
\hline
\end{tabular}


SILVA, M. G.; SANTOS, A. T.; FERNANDES, J. M.; GOMES, R. A. N.; NERI, M. A. T.; DINIZ, J. H.; JAREK, A.; ELVIDIO, G. LOCALIZACCÃO DE DANOS EM VIGAS DE CONCRETO ARMADO CONSIDERANDO O COMPORTAMENTO DOS MODOS DE VIBRAÇÃO. $4^{\circ}$ Simpósio Paranaense de Patologia das Construções (40 SPPC), artigo 4SPPC121, pp. 190 - 201, 2019. DOI: $10.4322 / 2526-7248.041$

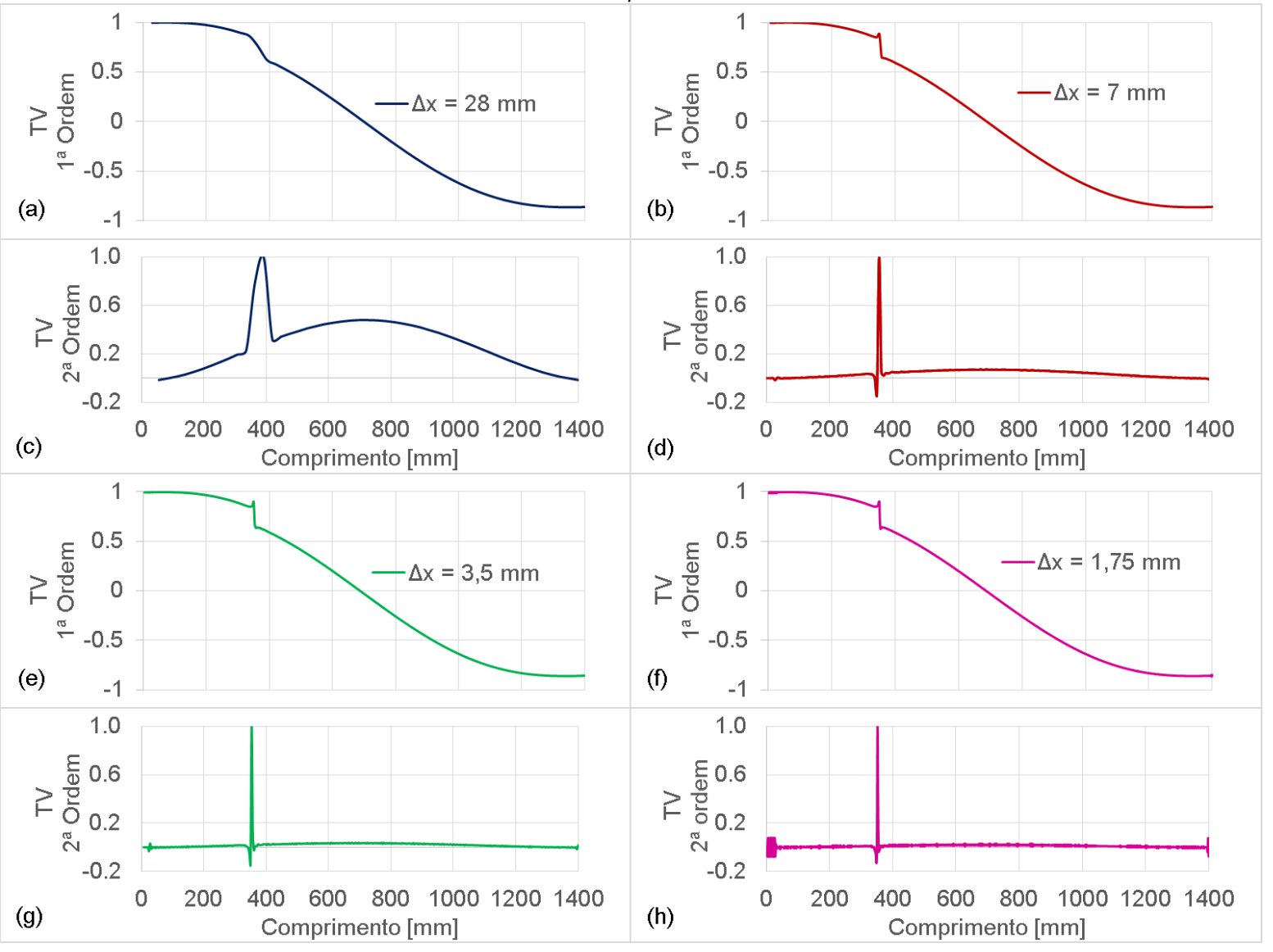

Figura 6: Localização do dano a partir das taxas de variação de $1^{\text {a }}((\mathrm{a}),(\mathrm{b}),(\mathrm{e})$ \& (f)) e $2^{a}((c),(d),(g) \&(h))$ ordens, do primeiro modo de vibração, para resoluções com $\Delta x$ de $28 \mathrm{~mm}, 7 \mathrm{~mm}, 3,75 \mathrm{~mm}$ e $1,75 \mathrm{~mm}$ no caso 2 de deterioração $(A=350 \mathrm{~mm})$.

\section{Considerações finais}

Notou-se que, para os danos propostos no presente estudo, a correlação do coeficiente MAC da viga analisada se manteve elevada - da ordem de $98 \%$ ou superior. Assim, não foi possível prever a existência de falhas (e posteriormente a redução de rigidez associada) em uma estrutura considerando apenas o referido índice, uma vez que em 100\% o mesmo indica correlação completa entre os dados analisados.

Por outro lado, foi possível identificar que o coeficiente COMAC é uma maneira eficaz para detecção da localização de danos em estruturas de concreto armado. Foi noticiado, por meio dos resultados obtidos, que é o comportamento da curva do coeficiente COMAC que indica tal posição e não as regiões de maio redução do coeficiente, como se tem proposto na literatura [5-7]. Nesse sentido, quanto melhor discretizado é o autovetor do coeficiente, mais precisos são os resultados encontrados - como foi apresentado no presente estudo.

Como os danos são localizados com base nas regiões de descontinuidade da curva COMAC, foram empregadas análises de taxa de variação secante como pósprocessamento dos dados obtidos. Essas análises, principalmente para o emprego da taxa de variação em segunda ordem, apresentaram maior precisão na identificação do dano nos casos considerados. Desse modo, fica demonstrada a 
SILVA, M. G.; SANTOS, A. T.; FERNANDES, J. M.; GOMES, R. A. N.; NERI, M. A. T.; DINIZ, J. H.; JAREK, A.; ELVIDIO, G. LOCALIZAÇÃO DE DANOS EM VIGAS DE CONCRETO ARMADO CONSIDERANDO O COMPORTAMENTO DOS MODOS DE

VIBRAÇÃO. 40 Simpósio Paranaense de Patologia das Construções (40 SPPC), artigo 4SPPC121, pp. 190 - 201, 2019. DOI: $10.4322 / 2526-7248.041$

importância de a definição do coeficiente COMAC não ser o último passo na identificação de regiões danificadas em estruturas de concreto armado com base nos modos de vibração da mesma.

Por fim, foram aplicadas as análises de taxa de variação secante diretamente sobre o primeiro modo transversal de vibração, sem considerar o comportamento dos coeficientes COMAC. Além dessa alternativa de análise não requerer informações da estrutura no seu estado íntegro para comparação dos dados e consequente localização do dano, foi notado ainda um aumento na precisão dos resultados obtidos. Por outro lado, como são utilizados dados de apenas um modo natural de vibração, a possibilidade de o dano estar posicionado sobre o respectivo nó modal pode levar a graves erros nessa condição de análise, ressaltando a importância da utilização do coeficiente COMAC.

Apesar de terem sido obtidos bons resultados computacionais para localização do dano com o emprego do índice COMAC, aplicar essa técnica experimentalmente é um desafio. Isso se deve à necessidade de ter uma grande quantidade de dados para formar os autovetores dos modos de vibração com satisfatória discretização (sendo adotado um espaçamento, para resultados aceitáveis, da ordem de $1 \mathrm{~cm}$ no presente estudo). Em ensaios in-loco isso significa o emprego de grande quantidade de acelerômetros para compor os modos de vibração da estrutura, o que se torna inviável, ou ainda a utilização de técnicas alternativas para a obtenção desse parâmetro modal.

\section{Agradecimentos}

Os autores agradecem ao Lactec, ao Grupo Neoenergia, à COSERN (Companhia Energética do Rio Grande do Norte) e ao PPGECC-UFPR (Programa de PósGradação em Engenharia de Construção Civil) pela oportunidade de realizar tal estudo dentro do projeto de pesquisa intitulado "Avaliação da integridade estrutural de postes de distribuição por meio de monitoramentos dinâmicos".

\section{Referências}

[1] Farrar, C. R.; Worden, K. (2007) An introduction to structural health monitoring. Philosophical Transactions of the Royal Society A, 365, 303-315. University of Sheffield, Sheffield, Inglaterra.

[2] Ewins, D. J. (2000) Modal Testing: Theory, Practice and Application. 2nd ed. Research studies press LTD. Hertfordshire, Inglaterra.

[3] Farrar, C. R.; Doebling, S. W.; Nix, D. A. (2001) Vibration-based structural damage identification. Philosophical transactions: mathematical, physical and engineering sciences, 359, 1778, 131-149. Royal Society.

[4] Erazo, K.; Sen, D.; Nagarajaiah, S.; Sun, L. (2019) Vibration-based structural health monitoring under changing environmental conditions using Kalman Filtering. Mechanical systems and signal processing, 117, 1-15. 
SILVA, M. G.; SANTOS, A. T.; FERNANDES, J. M.; GOMES, R. A. N.; NERI, M. A. T.; DINIZ, J. H.; JAREK, A.; ELVIDIO, G. LOCALIZAÇÃO DE DANOS EM VIGAS DE CONCRETO ARMADO CONSIDERANDO O COMPORTAMENTO DOS MODOS DE VIBRAÇÃO. 40 Simpósio Paranaense de Patologia das Construções (40 SPPC), artigo 4SPPC121, pp. 190 - 201, 2019.

[5] Genovese, M.; Oliveira, A. B. S.; Doz, G. N.; Brito, J. L. V. (2009) Análise experimental para avaliação da integridade estrutural por meio das alterações nas características dinâmicas. XXIX Jornadas Sudamericanas de Ingenieria Estructural. Universidade de Brasília, Departamento de Engenharia Civil e Ambiental, Brasília, DF, Brasil.

[6] Zhou, Z. (2006) Vibration-based damage detection of simple bridge superstructures. Tese (doutorado), University of Saskatchewan, Saskatoon, Canadá.

[7] Ndambi, J. M.; Vantomme, J.; Harri, K. (2002) Damage assessment in reinforced concrete beams using eigenfrequencies and mode shape derivatives. Engineering Structures, 24, 501-515.

[8] Larson, R.; Edwards, B. H. (2005) Cálculo com aplicações. 6th Ed. Universidade da Florida, Florida, Estados Unidos.

[9] Blank, B. E.; Krantz S. G. (2011) Calculus single variable. 2nd Ed. Universidade de Washington, St. Louis, Estados Unidos.

[10] Sevim, B.; Bayraktar, A.; Altunisik, A. C.; Atamturktur, S.; Birinci, F. (2011) Finite element model calibration effects on the earthquake response of masonry arch bridges. Finite elements in analysis and design, 47, 621-634.

[11] Mechanical APDL theory reference. (2019) SAS IP, Inc., Release 17.0. Disponível <https://www.sharcnet.ca/Software/Ansys/17.0/enus/help/ans_thry/ansys.theory.html>. Acesso: 11 jun.

[12] Grimes, R. G.; Lewis, J.G.; Simon, H. D. (1996) A Shifted Lanczos Algorithm for Solving Sparse Symmetric Generalized Eigenproblems. SIAM Journal Matrix Analysis Applications. 15, 1, 228-272.

[13] Jarek, A.; Santos, A. T.; Gavassoni, E.; Lobo, L. A.; Silva, M. G.; Lacerda, L. A. (2019) Avaliação da integridade estrutural de postes de distribuição por meio de monitoramentos dinâmicos - Modelagem matemática, dinâmica de postes. Institutos Lactec, Paraná, Brasil. 